\title{
L'exil intérieur des Québécois dans le Canada de la Charte
}

\section{Guy Laforest*}

\section{Introduction}

Je vais commencer cet article par une note personnelle. Il y a vingt-cinq ans, au temps de l'entrée en vigueur de la Charte canadienne des droits et libertés ${ }^{1}$, je vivais à Montréal et j'étudiais à l'Université McGill. Parmi mes professeurs, il y avait deux grands intellectuels qui étaient aussi deux grands idéalistes, Charles Taylor et James Tully ${ }^{2}$. J'ai beaucoup appris d'eux et avec le temps, ils sont devenus des amis. J'avais d'autres professeurs qui m'ont influencé, peut-être moins directement, mais tout aussi durablement, notamment les Blema Steinberg, Daniel Latouche, James Mallory et Harold Waller. Leur approche était teintée de réalisme, et elle contrebalançait à merveille celle que je trouvais chez Taylor et Tully. En philosophie, l'approche réaliste est celle du libéralisme sans illusions que l'on trouve chez les Judith Shklar, Raymond Aron, Isaiah Berlin et Karl Popper, selon laquelle en politique, il faut d'abord et avant tout éviter le pire. Il faut entendre par là la cruauté, l'effroi, la terreur, la violence, tout ce qui peut broyer la personne humaine, l'atteindre dans sa dignité et dans son intimité. A ce titre je partage le jugement d'Irvin Studin qui écrivait récemment que le Canada est un formidable succès à l'échelle de l'humanité, l'un des pays parmi les plus " pacifiques, justes et civilisés ». Un pays où, pour ajouter ma propre voix, les forts comme les faibles peuvent dormir tranquilles dans un milieu social humain, décent, confortable, sans craindre le pire. Tout cela compte pour beaucoup dans l'histoire de l'humanité.
J'utilise ce ton pour montrer un certain sens des proportions dans les analyses que je vais développer sur l'exil intérieur des Québécois dans le Canada de la Charte. Comme pas mal d'autres personnes au Québec, sur les plans de l'identité politique et de l'appartenance, je ne suis pas un citoyen heureux dans le Canada de la Charte 4 . Au-delà de mes sentiments personnels, je crois que cela s'explique par le fait que le Québec n'est pas intégré correctement dans le nouveau Canada qui a surgi depuis la réforme constitutionnelle de 1982. Paradoxalement, cette réforme a vu le jour, pour une bonne part, à cause du dynamisme et des pressions exercées par le Québec sur le Canada dans la foulée de la Révolution tranquille. Au lieu d'améliorer la situation, la réforme de 1982 l'a aggravée. Telle est la thèse que je promouvrai dans cet article. L'expression "exil intérieur" décrit très bien le fondement de ma pensée. Car un exilé de l'intérieur, c'est quelqu'un qui se sent inconfortable, qui vit comme un étranger au sein de son propre pays.

\section{Pierre Trudeau, l'exil des Québécois et la Charte}

\footnotetext{
Mais c'est encore une fois la tendance toujours accentuée, c'est le poids dans la balance du côté du provincialisme aux dépens d'une institution fédérale, ou d'une législation, qui, jusqu'à présent, donnait aux Canadiens un sens d'appartenance nationale, un peu comme la Charte des droits et libertés était impor-
} 
tante pour l'unité canadienne, un peu comme le rapatriement de la Constitution, un peu comme le drapeau canadien. Tout cela, c'est important en ce sens que ça fait comprendre aux Canadiens qu'ils partagent avec TOUS les Canadiens, de TOUT le pays, un MÊME ensemble de valeurs fondamentales ${ }^{5}$.

La question de l'exil intérieur des Québécois est rendue immensément plus complexe si l'on y ajoute le rôle prépondérant qu'y a joué Pierre Elliott Trudeau, sans conteste l'une des plus grandes personnalités politiques du Québec et du Canada au XXe siècle. Sur les plans de l'appartenance et de l'identité, les Québécois ont été en quelque sorte mis en exil par l'un des leurs. Le fédéralisme a occupé une place importante dans la vie de M. Trudeau. Sauf qu'un examen de ses actions et de ses écrits dans les années quatre-vingt, replacé sur l'horizon d'ensemble de sa vie politico-intellectuelle, révèle en lui un nationaliste et un souverainiste canadien bien plus qu'un fédéraliste. C'est très clairement ce qui ressort du très important livre qu'André Burelle, philosophe et ancien rédacteur des discours de M. Trudeau, vient de consacrer à son œuvre $^{6}$. J'y reviendrai dans un moment. Au soir de sa carrière, M. Trudeau rêvait d'établir une fois pour toutes la souveraineté de la nation canadienne et celle du gouvernement central. Dans le débat sur l'Accord du lac Meech ${ }^{7}$, il se demanda souvent «comment peut-on rendre un pays plus fort en affaiblissant le seul gouvernement capable d'exprimer le point de vue de tous les Canadiens ${ }^{8}$. Plutôt que de chercher à trouver dans le fédéralisme et ses institutions un équilibre entre un projet national canadien et un projet national québécois, il résolut après le référendum de 1980 à recourir au nationalisme canadien pour changer le pays et l'emporter définitivement contre les souverainistes québécois'. La Charte fut l'instrument d'un tel dessein, comme le politologue Alan Cairns l'a rappelé dans un entretien avec l'historien Robert Bothwell :

The prime one, the obvious one, is what the Charter appears to be on its face, a way of protecting citizen rights against the state. From Trudeau's perspective, however, the much more important goal was the attempt to generate a national identity, and this really meant an attack on provincialism. It was a way of trying to get Canadians to think of themselves as possessors of a common body of rights independent of geographical location, which would constitute a lens through which they would then view what all governments were doing. So it was really a de-provincializing strategy, primarily aimed at Quebec nationalism, but also at the general centrifugal pressures that were developing across the federal system ${ }^{10}$.

Dans son livre de 2005, André Burelle explique lucidement les péripéties politiques qui ont amené M. Trudeau en 1980 à rompre le délicat équilibre qui prévalait jusqu'alors dans son esprit entre le personnalisme communautaire de l'époque citélibriste - la pensée des Jacques Maritain et Emmanuel Mounier - réconciliable avec le fédéralisme de 1867 et une certaine acceptation de la différence nationale québécoise, et un libéralisme ultra-individualiste et symétrique faisant l'affaire du "nation-building » canadien $^{11}$. Dans l'esprit de Burelle, le fédéralisme "one nation" de la Loi constitutionnelle de $1982^{12}$ s'appuie sur l'unitarisme républicain et part des prémisses d'un libéralisme individualiste et anti-communautaire. Selon lui, cela s'opérationnalise de la manière suivante :

a) Tous les individus y sont fondus en une seule nation civique qui délègue au Parlement fédéral la totalité de sa souveraineté nationale;

b) Investi de cette souveraineté, le Parlement central confie aux provinces les pouvoirs fonctionnellement mieux exercés par elles ${ }^{13}$.

Quelle sorte de fédéralisme découle d'une telle logique? Selon Burelle, c'est un fédéralisme qui ne respecte plus la différence québécoise, qui fait fi du principe de non-subordination entre deux ordres de gouvernement souverains dans leurs compétences respectives, qui s'éloigne autrement dit de ce que les fondateurs de la fédération canadienne avaient voulu faire en 1867. Selon Burelle, l'esprit de 1982 bafoue celui de 1867 par l'opération entrecroisée des principes suivants :

a) la pratique d'une subsidiarité dévoyée i.e. d'une dévolution de souveraineté descen- 
dante (top down) qui part de l'État central;

b) l'existence d'un gouvernement senior «national » et de gouvernements juniors « provinciaux »;

c) l'attribution à Ottawa d'un droit d'ingérence pour garantir "l'intérêt national» dans les champs de compétence provinciale;

d) l'identité de droit et de traitement des individus et des provinces vu leur fusion au sein d'une seule et même nation républicaine ${ }^{14}$.

Dans l'ensemble, l'interprétation de Burelle me semble assez juste. Il avait dit cela autrement dans son livre de 1994, en parlant d'un gouvernement par les juges via une "Charte nationale » et d'un gouvernement par le " peuple canadien » permettant à Ottawa de contourner le partage des compétences. ${ }^{15}$ J'y apporterais toutefois une nuance : il faut faire une distinction entre la vision de la Charte promue par M. Trudeau luimême et le contenu réel de celle-ci. Je pense que Burelle comprend bien Pierre Trudeau. Toutefois, comme la juge-en-chef de la Cour suprême, Beverley McLachlin, le fait bien ressortir dans un discours que je citerai plus loin dans cet article, les catégories de la Charte ne sont pas restreintes aux seuls droits individuels. Il y a de la place dans la Charte pour le patrimoine multiculturel des Canadiens et pour les droits collectifs des peuples autochtones. Toutefois, il n'y en a pas pour l'idée de la différence québécoise et pour le principe que devraient en découler des conséquences juridiques. Avant d'explorer dans la prochaine section des moyens qui pourraient mettre fin à l'exil intérieur des Québécois dans le Canada de la Charte, je vais parachever celleci avec deux analyses de l'esprit de 1982 que l'on doit à Eugénie Brouillet et José Woehrling, deux des meilleurs professeurs de droit public dans le Québec d'aujourd'hui :

c'est précisément en raison du potentiel intégrateur de la charte canadienne que le gouvernement fédéral en a fait la pierre angulaire de la réforme constitutionnelle de 1982. Sur le plan politique, la reconnaissance de droits et libertés supralégislatifs à chacun des citoyens canadiens constituerait le fondement de leur identité commune et renforcerait ainsi l'unité de la nation canadienne. Sur le plan juridique, le transfert entre les mains des tribunaux du pouvoir de mise en ouvre de ces droits et libertés et le développement de normes nationales positives et négatives qui en découleraient joueraient dans un sens centralisateur ${ }^{16}$.

Dans la mesure où la protection des droits par un instrument constitutionnel est un dispositif antimajoritaire, elle vient limiter l'autonomie politique des minorités qui disposent d'une ou de plusieurs entités territoriales. La minorité qui contrôle une telle entité voit son pouvoir politique limité au profit de ses propres minorités et de ses propres membres... La majorité au niveau national peut alors céder à la tentation d'utiliser son pouvoir pour imposer à sa minorité le respect de garanties excessives au profit de "la minorité dans la minorité ». On a l'impression, parfois, que le groupe majoritaire au niveau national défend ses propres intérêts sous le prétexte des droits de la personne et des droits des minorités ${ }^{17}$.

\section{Pour mettre fin à l'exil}

La situation politique est en flux au Canada et au Québec à l'automne 2007, avec la présence de deux gouvernements minoritaires, et tout porte à croire qu'elle continuera de l'être au cours des prochains mois. C'est ce que j'appelle une équation du deuxième degré à plusieurs inconnues, installant du brouillard dans la tête de la plupart des experts. Il faut par ailleurs clairement reconnaître plusieurs développements positifs des dernières années: l'entente asymétrique Canada-Québec sur la santé signée en 2004 par les gouvernements Martin et Charest ${ }^{18}$, la formulation de la doctrine du fédéralisme d'ouverture par Stephen Harper lors de la campagne électorale qui a mené à l'élection d'un gouvernement conservateur à Ottawa en janvier 2006, l'entente Harper-Charest de mai 2006 acceptant le principe d'un rôle international particulier pour le Québec et intégrant un représentant du gouvernement du Québec dans la délégation canadienne à l'Unesco ${ }^{19}$, la résolution de la Chambre des communes de novembre 2006 stipulant que les Québécois forment une nation au sein d'un Canada uni - malgré les ambiguités qui perdurent entre les versions française et anglaise du texte ${ }^{20}$, enfin la prise en compte du problème du déséquilibre fiscal dans le budget fédéral en février $2007^{21}$. Par ailleurs, il se pourrait que 
le gouvernement Harper bouge dans la direction d'un certain encadrement du pouvoir fédéral de dépenser dans le discours du Trône prévu pour le 16 octobre $2007^{22}$. Dans ma perspective, il est incontestable que ces développements contribuent à atténuer le malaise, à changer le climat dans lequel opère notre vie politique. Ils ne réussissent cependant pas à prendre en compte le problème de la place du Québec dans l'architecture constitutionnelle du Canada. Pour progresser dans cette direction, il faudra un jour amender la Loi constitutionnelle de 1982 et la Charte canadienne des droits et libertés.

L'article premier de la Charte stipule que les droits inclus dans le texte "ne peuvent être restreints que par une règle de droit, dans des limites qui soient raisonnables et dont la justification puisse se démontrer dans une société libre et démocratique $"^{23}$. Dans la mesure où, comme la Cour suprême l'a d'ailleurs reconnu en en faisant le premier des piliers normatifs de notre édifice constitutionnel dans le Renvoi sur la sécession du Québec, le fédéralisme est un principe structurant de la communauté politique canadienne, l'article premier de la Charte devrait référer à une "fédération libre et démocratique " plutôt qu'à une "société libre et démocratique ». Ce tout petit changement aurait deux effets majeurs: tout d'abord, il instruirait les juges de la nécessité de prendre en compte le caractère névralgique du principe fédéral dans leur compréhension des règles juridiques de notre régime; ensuite, cela aurait une valeur pédagogique, dans la mesure où on inviterait les citoyens à mieux comprendre l'importance du fédéralisme dans l'identité politique canadienne. En prime, comme conséquence, les Québécois se sentiraient moins seuls à prendre au sérieux le fédéralisme dans la compréhension du Canada!

Le deuxième changement que j'ai en tête découle logiquement du débat qui a marqué la vie publique canadienne à l'automne 2006 à propos de l'opportunité de reconnaître le fait national québécois. Lancé par Michael Ignatieff lors de la course à la direction du Parti libéral du Canada, le débat s'est achevé par la motion de reconnaissance présentée par le Premier Ministre Harper à la Chambre des Communes. Pour mettre fin à l'exil intérieur des Québécois, il faut placer leur manière de se définir dans un texte qui compte vraiment pour les Canadiens. Et ce texte, c'est la Charte canadienne des droits et libertés. On pourrait ainsi ajouter la sous-section I.A à la Charte, reconnaissant que le Québec forme au Canada une société nationale distincte, stipulant aussi que le gouvernement et l'Assemblée Nationale du Québec ont l'obligation de protéger et promouvoir une telle société. C'était l'expression préférée par Claude Ryan et par André Laurendeau il y a quarante ans, au temps de la Commission royale d'enquête sur le bilinguisme et le biculturalisme. Certains y devineront le fantôme de la clause de la société distincte au temps de l'Accord du lac Meech. Ils ne s'y tromperont pas. Beaucoup de Québécois ont vu dans la réforme constitutionnelle de 1982, non sans raisons sur la base de la section précédente de ce texte, une tentative pour créer une seule et grande nation canadienne subsumant toutes les autres appartenances et en particulier celle découlant du nationalisme québécois moderne. L'adoption de l'Accord du lac Meech aurait corrigé le tir à cet égard. Un jour ou l'autre, cette question va resurgir et elle fera partie de la doctrine constitutionnelle d'un parti politique gouvernant le Québec. Il est intéressant de rappeler, sur cette question, les propos de l'actuel chef du Parti libéral du Canada, M. Stéphane Dion, revenant alors qu'il était ministre dans le gouvernement Chrétien en 1996 sur l'enjeu de la société distincte et de sa reconnaissance :

Quelle est l'essence de la disposition sur la société distincte ? Cette disposition serait un article d'interprétation, semblable à l'article 27 de la Charte des droits et libertés, qui reconnaît le multiculturalisme. Elle garantit que, dans les zones grises de la Constitution, dans les domaines où il faut interpréter les règles, la Cour suprême tiendra compte du caractère distinct du Québec dans des domaines comme la langue, la culture et le droit civil. Ce sera une clarification utile, mais qui ne modifiera en rien le partage des pouvoirs prévu par la Constitution. Il ne s'agit pas d'une demande de statut spécial ni de privilèges particuliers ${ }^{24}$.

Dès l'époque du régime britannique, à la fin du XVIIIe siècle, on s'est refusé à appliquer la politique d'assimilation et d'homogénéisation qui a dominé assez généralement la période de la consolidation de l'État-nation moderne en Eu- 
rope. L'Acte de Québec de 1774 garantissait aux nouveaux sujets d'origine française de sa Majesté des libertés religieuses et le maintien de leur droit civil $^{25}$. Quand le Canada est devenu un Dominion fédéral en 1867, un tel esprit d'ouverture, de reconnaissance de la diversité et de respect des droits des minorités s'est exprimé dans l'attribution aux provinces (c'était clairement une revendication du Canada-Est, l'ancienne appellation du Québec, mais pas uniquement de lui) de la juridiction sur la propriété et les droits civils, ainsi que dans les dispositions protégeant les minorités religieuses. On peut d'ailleurs retrouver les origines historiques et juridiques du fédéralisme asymétrique au Canada dans la formulation de l'article 94 de la Loi constitutionnelle de $1867^{26}$, lequel omet le Québec du périmètre de validité des pratiques d'homogénéisation en matière de propriété et de droits civils entre le gouvernement fédéral et les provinces de common law ${ }^{27}$. Cette originalité canadienne dans l'histoire de l'Etat-nation moderne s'est notamment exprimée en 1982 dans la rédaction de l'article 27 de la Charte, lequel stipule que «Toute interprétation de la présente charte doit concorder avec l'objectif de promouvoir le maintien et la valorisation du patrimoine multiculturel des Canadiens». Le troisième changement que j'estime nécessaire pour mettre un terme à l'exil intérieur des Québécois dans le Canada de la Charte porte sur cet article.

Si le débat sur la nation québécoise a soulevé pas mal d'intérêt dans les médias aussi bien anglophones que francophones du pays en 2006, on peut en dire autant, dans le Québec de l'automne 2007, pour les travaux de la Commission de consultation sur les pratiques d'accommodement reliées aux différences culturelles (Commission co-dirigée par Gérard Bouchard et Charles Taylor). Bien que l'on ne puisse préjuger de l'orientation générale du rapport, le document préliminaire de consultation publié par la Commission laisse clairement entendre que celle-ci a choisi de donner une interprétation large à son mandat, acceptant donc de se pencher sur la question des rapports entre majorité et minorités ${ }^{28}$. Le Canada et le Québec sont des terres d'immigration. Cette histoire s'est accélérée dans la deuxième moitié du vingtième siècle et cela ne va pas changer. J'estime que ce phénomène social est un bien et que cela honore tous les gens qui vivent au Canada et qui ont à confronter ce monde tourmenté qui est le nôtre. Mais j'estime aussi que dans son portrait des droits et obligations qui découlent de son article 27 sur le patrimoine multiculturel des Canadiens, la Charte omet de préciser une réalité fondamentale : ce patrimoine s'incarne au Canada dans les réseaux institutionnels de deux sociétés d'accueil, dont l'une au Québec décline sa vie sociale dans le respect du principe de la prépondérance de la langue française. Une telle précision m’apparaît essentielle pour l'intégration à la fois juste et stable du Québec dans le Canada d'aujourd'hui.

Le Canada est une fédération libre et démocratique intégrant la société nationale distincte du Québec et un patrimoine multiculturel incarné dans deux sociétés d'accueil, dont l'une vit la modernité principalement en français. C'est ce qui manque à la Charte canadienne des droits et

libertés, si je comprends bien la situation, pour aller au-delà de l'aliénation politique des Québécois. De telles transformations ne mettraient pas fin pour toujours aux conflits politiques dans le régime fédéral canadien, y compris entre le Québec et ses partenaires. Celles et ceux qui veulent mettre fin une fois pour toutes aux "chicanes politico-constitutionnelles» négligent le fait que la vie politique sera toujours affaire de dialogues et de débats pouvant déboucher sur des affrontements. Toutefois, ces changements auraient pour effet de guérir une blessure et de redonner confiance dans le droit et dans les institutions canadiennes. Par ailleurs, l'angle d'analyse choisi dans cet article est bien loin d'épuiser la totalité du réel, je l'admets bien volontiers. Comme les Catalans et comme plusieurs États fédérés dans le monde, les Québécois pourraient faire preuve aussi d'audace et d'imagination en se dotant, d'une manière autonome, d'une constitution interne renforçant l'épine dorsale des institutions de leur société. ${ }^{29}$ Enfin, des Québécois plus confiants dans leur avenir et mieux intégrés devraient se montrer plus ouverts aux propres désirs de changement des autres Canadiens, lesquels sont certainement aussi légitimes que les leurs. Des Québécois qui ne seraient plus des exilés de l'intérieur devraient être capables de proclamer, à leur manière, leur allégeance envers le Canada, et de s'engager dans des projets communs pour le XXIe siècle. 


\section{Conclusion}

Dans le contexte de la commémoration du $25^{\mathrm{e}}$ anniversaire de l'adoption de la Charte canadienne des droits et libertés, je chercherai une dernière fois à faire comprendre cette notion d'exil intérieur des Québécois en rappelant un autre événement ramené à notre mémoire en 2007, le célèbre cri du général Charles de Gaulle au balcon de l'hôtel-de-ville de Montréal en juillet 1967 «Vive le Québec libre! ». Il y a lieu d'être un peu nostalgique lorsque l'on se remémore l'année 1967. C'était l'ère du Centenaire de la Confédération canadienne et celle de l'Exposition universelle de Montréal, une époque où les gens d'ici et d'ailleurs étaient invités à vivre d'espoir et d'idéalisme à la hauteur du lyrisme de la "Terre des Hommes » de St-Exupéry. Il me semble que, si le discours du général de Gaulle a eu un tel retentissement à Montréal en 1967, c'est qu'il répondait à trois aspirations profondes de la société québécoise : il affirmait solennellement le droit à la différence du Québec au Canada et en Amérique, il donnait fortement le goût d'une liberté politique qui peut prendre plusieurs formes dans la modernité, et enfin, il répondait à une soif d'universel, à un immense désir de reconnaissance. En 1967, Charles de Gaulle a donné une dimension planétaire à la question du Québec. Quand on se met à cerner la signification profonde de la Charte des droits et libertés pour le Canada, on s'aperçoit qu'elle a conforté des aspirations semblables pour le nationalisme majoritaire des Canadiens vivant à l'extérieur du Québec, de même que pour une minorité importante à l'intérieur du Québec. En proposant un équilibre intelligent entre des droits individuels, des droits collectifs et des droits pour des personnes rattachées à des communautés minoritaires, et en faisant cela de manière originale par rapport au modèle du Bill of Rights américain, la Charte a représenté une forte affirmation de la différence canadienne parmi les démocraties libérales occidentales. Deuxièmement, la Charte a contribué à parachever sur l'axe de la liberté politique l'indépendance complète de l'État-nation canadien. Finalement, la Charte et son rayonnement dans le monde ont nourri le désir de reconnaissance des Canadiens, en faisant de leur pays l'avant-garde d'une civilisation de la différence pour le XXIe siècle. C'est ce que l'on peut deviner en lisant la prose des meilleurs penseurs de l'école idéaliste canadienne, de Charles Taylor à James Tully, en passant par Will Kymlicka, John Ralston Saul et Michael Ignatieff. La juge-en-chef de la Cour suprême du Canada, Beverley Mclachlin, s'inscrivait dans cette tradition dans un discours prononcé le 17 avril 2002, à l'occasion du $20^{\mathrm{e}}$ anniversaire de l'entrée en vigueur de la Charte :

Nous avons une Charte qui reflète nos valeurs les plus fondamentales et nous dit qui nous sommes comme peuple et ce à quoi nous tenons. Nous avons une Charte qui suscite l'admiration du monde entier. Enfin, fait plus important encore, nous avons une Charte que les Canadiens et les Canadiennes ont fait leur au cours des deux dernières décennies. La Charte, c'est à nous. La Charte : c'est nous ${ }^{30}$.

On peut comprendre alors que la Charte soit un motif de grande fierté au Canada, que beaucoup de gens aient le goût de dire, comme la juge-en-chef en 2002, la Charte c'est à nous et la Charte c'est nous. Sauf que, vu du Québec, cette Charte a été adoptée de façon antidémocratique sans notre consentement, au mépris de l'opposition de notre gouvernement et de l'Assemblée Nationale, atteignant ainsi le peuple du Québec lui-même pour reprendre les paroles de Claude Ryan à l'époque ${ }^{31}$. L'autonomie législative du Québec en matières linguistiques a été réduite, le principe fédéral a été affaibli dans nos institutions et dans notre culture politique, le droit à la différence du Québec n'a pas été intégré à la Charte. Plus précisément, le Canada de 1982 et de la Charte ne reconnaît explicitement ni la différence québécoise, ni le fait que cette différence devrait entraîner des conséquences politiques et juridiques. L'idéalisme canadien, qui voit dans notre système politique et surtout dans la Charte un exemple porteur pour l'humanité dans son ensemble, me semble condamné à rester profondément inauthentique tant que l'on n'aura pas trouvé une façon juste et raisonnable de reconnaître la différence québécoise, dans le droit aussi bien que dans les symboles $^{32}$. Sans pouvoir traiter ici de tous les aspects de cette question, il me semble évident que les Québécois ont choisi de vivre leur quête d'identité et de liberté dans le Canada. Le Québec est dans le Canada pour y rester, sauf qu'il s'y reconnaît trop imparfaitement dans ses institutions. 
Un grand esprit de McGill et du Québec, Charles Taylor, soulignait naguère, dans une réflexion sur le nationalisme québécois, qu'il faut être impitoyable envers nos mythes essentialistes. Le nationalisme majoritaire canadien s'enferme dans un mythe essentialiste semblable lorsqu'il s'imagine que l'on peut en ce pays occulter la profonde aliénation politique, l'exil intérieur des Québécois. Un autre grand esprit de l'humanisme occidental, Paul Ricoeur, a écrit que la mémoire historique des peuples doit être juste et heureuse. L'exil intérieur des Québécois est un obstacle fondamental au développement d'une mémoire historique juste et heureuse au Canada.

\section{Notes}

* Guy Laforest, Département de science politique, Université Laval, Québec, Québec, G1K 7P4, Guy.Laforest@pol.ulaval.ca.

$1 \quad$ Charte canadienne des droits et libertés, partie I de la Loi constitutionelle de 1982, constituant l'annexe B de la Loi de 1982 sur le Canada (R.U.), 1982, c.11 (LLJCan).

2 On trouvera des analyses de la situation canadienne actuelle inspirées par les travaux de Taylor et Tully dans Alain-G. Gagnon, Au-delà de la nation unificatrice: plaidoyer pour le fédéralisme multinational, Barcelone et Montréal, Institut d'estudis autonomics et Boréal, 2007; voir aussi Guy Laforest, Pour la liberté d'une société distincte, Québec, Presses de l’Université Laval, 2004.

3 Irvin Studin, ed., What is a Canadian? FortyThree Thought-Provoking Responses, Toronto, McClelland \& Stewart, 2006 à la p. 184.

4 Ma thèse est aussi celle d'André Burelle, Le mal canadien, Montréal, Fides, 1994; voir aussi Christian Dufour, Lettre aux souverainistes québécois et aux fédéralistes canadiens qui sont restés fidèles au Québec, Montréal, Stanké, 2000.

5 Pierre-Elliott Trudeau, « Où est notre patriotisme national? » dans Donald Johnston, dir., Lac Meech : Trudeau parle, Montréal, Hurtubise HMH, 1988 à la p. 41.

6 André Burelle, Pierre-Elliott Trudeau : l'intellectuel et le politique, Montréal, Fides, 2005.

7 (3 juin 1987) Consulté en ligne le 27 novembre 2007 à l'adresse suivante en ligne : L'Encyclopédie canadienne $<\mathrm{http} / / / \mathrm{www}$.thecanadianencyclopedia.com/index.cfm?PgNm=TCE\&Params $=\mathrm{f}$
1SEC861644>.

8 Pierre-Elliott Trudeau, « Nous, le peuple du Canada ", dans Donald Johnston, dir., Lac Meech : Trudeau parle, Montréal, Hurtubise HMH, 1988 à la p. 104.

9 J'ai beaucoup écrit sur ces questions. Voir notamment Guy Laforest, Trudeau et la fin d'un rêve canadien, Québec, Septentrion, 1992; voir aussi un chapitre intitulé « La vraie nature de la souveraineté : réponse à mes critiques à propos de Trudeau et la fin d'un rêve canadien ", dans Guy Laforest, Pour la liberté d'une société distincte, Québec, Presses de l'Université Laval, 2004 aux pp. 219-36.

10 Robert Bothwell, Canada and Quebec: One Country, Two Histories, Vancouver, UBC Press, 1995 à la p. 180.

11 Supra note 6 aux pp. 68-70.

12 Constituant l'annexe B de la Loi de 1982 sur le Canada (R.-U.), 1982, c.11 (LLJCan).

13 Supra note 6 à la p. 459.

14 Ibid. à la p. 459.

15 André Burelle, Le mal canadien, Montréal, Fides, 1994 à la p. 64.

16 Eugénie Brouillet, La négation de la nation: l'identité culturelle québécoise et le fédéralisme canadien, Québec, Septentrion, 2005 à la p. 325.

17 José Woehrling, «La Charte canadienne des droits et libertés et ses répercussions sur la vie politique ", dans Réjean Pelletier et Manon Tremblay, dirs., Le parlementarisme canadien, troisième édition revue et augmentée, Québec, Presses de l'Université Laval, 2005 à la p. 115.

18 (2004) Entente Canada-Québec sur la santé : Fédéralisme asymétrique qui respecte les compétences du Québec.

19 (2006) Accord Canada-Québec relatif à l'organisation des Nations Unies pour l'éducation, la science et la culture (UNESCO).

20 Débats de la Chambre des communes, 086 (24 novembre 2006).

21 Guy Laforest et Eric Montigny, dirs., « Le fédéralisme exécutif : problèmes et actualité », dans Réjean Pelletier et Manon Tremblay, Le parlementarisme canadien, troisième édition revue et augmentée, Québec, Presses de l'Université Laval, 2005 aux pp. 364-68; voir aussi Keith Banting et al., Open Federalism : Interpretations, Significance, Kingston, Institute of Intergovernmental Relations, Queen's University, 2006 (voir en particulier le chapitre signé par Alain Noël, «Il suffisait de presque rien : promises and pitfalls of open federalism, aux pp. 25-37); pour un portrait global voir Michael Murphy, dir., Canada : The State of the Federation 2005. Québec and Canada 
in the New Century: New Dynamics, New Opportunities, Montréal et Kingston, McGillQueen's University Press, 2007; et enfin voir Alain-G. Gagnon, dir., Le fédéralisme canadien contemporain : fondements, traditions, institutions, Montréal, Presses de l'Université de Montréal, 2006.

22 Débats de la Sénats, 1 (16 octobre 2007) aux pp. 1-7 (Son Excellence la Gouverneure générale).

23 Charte supra note 1, art. 1.

24 Stéphane Dion, « Notre pays est en danger », La Presse [de Montréal] (8 mars 1996) B7.

25 Pour une interprétation philosophique de cela, voir James Tully, Une étrange multiplicité : le constitutionnalisme à une époque de diversité, Québec, Presses de l’Université Laval, 1999 aux pp. 142-45.

26 (R.-U.), 30 \& 31 Vict., c. 3, reproduite dans L.R.C. 1985, app. II, n 5 (LLJCan).

27 Voir Janet Ajzenstat et al, Débats sur la fondation du Canada, trad. par Stéphane Kelly et Guy Laforest, Québec, Presses de l'Université Laval, 2004 aux pp. 336-37 et 378. On y trouve les discours de deux députés à l'Assemblée législative du Canada-Uni, M.C. Cameron et Christopher Dunkin, établissant clairement un lien entre l'article 94 et un statut asymétrique fort pour le Québec, tout cela ayant été négligé par plusieurs générations de constitutionnalistes québécois! Commission de consultation sur les pratiques d'accommodement reliées aux différences culturelles, Accommodements et différences: vers un terrain d'entente, la parole aux citoyens; Document de consultation, Gouvernement du Québec, 2007 à la p. v.

29 Guy Laforest, Pour la liberté d'une société distincte, Québec, Presses de L'Université Laval à la p. 256.

30 Beverley McLachlin, «En pleine maturité : l'identité nationale canadienne et la Charte des droits ", discours prononcé lors d'un colloque à Ottawa sur Les droits et les libertés au Canada, vingt ans après l'adoption de la Charte. Consulté en ligne le 27 novembre 2007 à l'adresse suivante : Cœur suprême du Canada < http://www. scc-csc.gc.ca/aboutcourt/judges/speeches/charter_f.asp $>$.

31 «Nous devons conclure avec beaucoup de fermeté que chaque fois que l'Assemblée nationale est atteinte dans ses prérogatives essentielles, c'est le peuple du Québec lui-même qui est atteint. Être indifférent à une atteinte faite aux pouvoirs de l'Assemblée nationale, c'est être indifférent ou traiter à la légère les aspirations et la réalité fondamentale du peuple québécois lui-même ».
Paroles d'un discours de Claude Ryan lors d'un débat à l'Assemblée nationale le 30 septembre 1981 dans : Réal Bélanger, Richard Jones et Marc Vallières, Les grands débats parlementaires 17921992, Québec, Presses de l'Université Laval, 1994 à la p. 39.

32 Christian Dufour, Lettre aux souverainistes québécois et aux fédéralistes qui sont restés fidèles au Québec, Montréal, Stanké, 2000 à la p. 114. 